\title{
Acoustical Source Tracing Using Independent Component Analysis and Correlation Analysis
}

\author{
Wei Cheng, Zhousuo Zhang, Jie Zhang, and Jiantao Lu \\ State Key Laboratory for Manufacturing System Engineering, Xian Jiaotong University, Xian 710049, China \\ Correspondence should be addressed to Wei Cheng; chengw@mail.xjtu.edu.cn
}

Received 4 January 2015; Accepted 25 February 2015

Academic Editor: Yumin He

Copyright ( 2015 Wei Cheng et al. This is an open access article distributed under the Creative Commons Attribution License, which permits unrestricted use, distribution, and reproduction in any medium, provided the original work is properly cited.

\begin{abstract}
Acoustical signals from mechanical systems reveal the operating conditions of mechanical components and thus benefit for machinery condition monitoring and fault diagnosis. However, the acoustical signals directly measured by the sensors in essential are the mixed signals of all the sources, and normally it is very difficult to be used for source identification or operating feature extraction. Therefore, this paper studies the acoustical source tracing problem using independent component analysis (ICA) and identifies the sources using correlation analysis: the measured acoustical signals are separated into independent components by independent component analysis method, and thus all the independent information of all the sources is obtained; these independent components are identified based on the prior information of the sources and correlation analysis. Therefore, all the source information contained in the measured acoustical signals can be independently separated and traced, which can provide more purer source information for condition monitoring and fault diagnosis.
\end{abstract}

\section{Introduction}

Vibration and acoustical signals caused by the collision and friction of mechanical components normally can reduce the operational precision and even shorten the service life of the machinery. However, the vibration and acoustical signals provide important information of the operating conditions, and thus machinery condition monitoring and fault diagnosis can be carried out based on a nondestructive measurement. As the measured acoustical signals are normally mixed signals of all the sources and noises, they have great significance to trace the source information from the measured signals and thus provide pure and independent information of mechanical components for an effective condition monitoring or fault diagnosis.

Generally, the acoustical signals directly measured by the sensors are complicated and rough information of the mechanical systems because they are mixed signals of all the sources and the transmission effects of the transmission paths. Recently, many researchers have devoted their efforts to transmission effects of vibration and acoustical signals. Xin et al. [1] investigated the vibroacoustic performance of a rectangular double-panel partition with enclosed air cavity and simply mounted on an infinite acoustic rigid baffle. Huang and Nutt [2] presented an analytical study of sound transmission through unbounded panels of functionally graded materials. Bravo et al. [3] analyzed the sound absorption and transmission properties of microperforated panels backed by an air cavity and a thin plate. Qian et al. [4] described an approach on the prediction of sound transmission loss for a finite sandwich panel with honeycomb core. Posson et al. [5] developed an analytical model for the sound transmission through an annular stator row in a configuration without mean flow. Lee and Kim [6] investigated the effects of structure on sound absorption and transmission loss of a composite sheet. Chandra et al. [7] analyzed the vibroacoustic and transmission loss characteristics of functionally graded material plates. All these articles studied the transmission characteristics of structures and can help to carry out a passive vibration and sound monitoring and control. However, normally it is a challenging task and costs plenty of time to build a precise model of acoustical transmission for complicated mechanical systems, and thus the engineering applications are limited.

To reveal the operating conditions of the mechanical components, signal processing methods are developed to extract the features hidden in the noisy response signals and 
thus benefit for system analysis, condition monitoring, or fault diagnosis. To clearly reveal the operating conditions of each mechanical component, blind source separation (BSS) and independent component analysis (ICA) are developed to separate the mixed signals into uncorrelated components without the knowledge of the sources and their mixing modes. Jutten and Herault [8] solved blind source separation problem with an adaptive algorithm based on neuromimetic architecture. Belouchrani et al. [9] proposed a technique to blindly separate the sources using high-order statistics. Comon [10] reexamined the concept of independent component analysis (ICA) and proposed an efficient ICA algorithm. Hyvärinen [11, 12] proposed a very famous ICA algorithm called fast-fixed algorithm for independent component analysis, which is very effective and efficient for linear systems. Since then, independent component analysis has attracted a worldwide attention and has been widely used in many engineering applications, such as image processing [13], EEG/MEG analysis [14], communications [15], radar [16], and modal parameter estimation [17]. In recent years, independent component analysis has been applied to mechanical signal processing, and some practical algorithms have been constructed. Antoni [18] addressed the issues of blind separation of vibration components. Cheng et al. studied the source number estimation [19], source separation [20], and source contribution evaluation [21] methods for mechanical systems based on an effective source separation. Henriquez et al. [22] provided a review of automatic fault diagnosis using independent component analysis and vibration signals. In this paper, independent component analysis is applied to acoustical source tracing (acoustical source separation and identification), and the separation performances of independent component analysis for acoustical signals are quantitatively evaluated by a numerical case study and an experimental study on a mechanical system with shell structures.

The remainder of this paper is organized as follows. In Section 2, basic theory and key principals of independent component analysis are introduced. In Section 3, the separation performance of the independent component analysis algorithm is tested by a numerical case study. In Section 4, the independent component analysis algorithm is applied to separate the acoustical signals, and then acoustical source tracing is carried out based on the source separation and correlation analysis. In Section 5, the conclusions of this study are summarized.

\section{Theory of Independent Component Analysis}

2.1. Principal Theory of Blind Source Separation. Blind source separation (BSS) including independent component analysis (ICA) is a powerful tool to separate a set of source signals $\mathbf{S}(t)=\left[\mathbf{s}_{1}(t), \mathbf{s}_{2}(t), \ldots, \mathbf{s}_{n}(t)\right]^{T}$ from the mixed signals $\mathbf{X}(t)=$ $\left[\mathbf{x}_{1}(t), \mathbf{x}_{2}(t), \ldots, \mathbf{x}_{m}(t)\right]^{T}$ without any information of the sources and their mixing approaches $[8,23,24]$, which can be described as follows:

$$
\begin{gathered}
\mathbf{x}_{i}(t)=\sum_{j=1}^{n} a_{i j} \mathbf{s}_{j}(t), \quad i=1,2, \ldots, m, j=1,2, \ldots, n, \\
\mathbf{X}=\mathbf{A S} \quad \text { Matrix form, }
\end{gathered}
$$

where $m$ is the number of the mixed signals, $n$ is the number of the sources, $\mathbf{x}_{i}$ is the $i$ th mixed signal collected at location $i, \mathbf{s}_{j}$ is the $j$ th source signal, $a_{i j}$ is a mixing coefficient, and $\mathbf{A}=\left\{a_{i j}\right\}$ is a mixing matrix.

The key process of BSS or ICA is to find a separating matrix $\mathbf{W}=\left[\boldsymbol{\omega}_{1}, \boldsymbol{\omega}_{2}, \ldots, \boldsymbol{\omega}_{n}\right]^{T}$ and recover the estimated sources $\mathbf{Y}(t)=\left[\mathbf{y}_{1}(t), \mathbf{y}_{2}(t), \ldots, \mathbf{y}_{n}(t)\right]^{T}$ by

$$
\mathbf{Y}=\mathbf{W X}=\mathbf{W}(\mathbf{A S})
$$

2.2. Measures of Independence. To effectively separate the mixed signals into independent components, some quantitative measures of the independence for a random variable should be developed. A well-known theory of independence is "non-Gaussian is independent," and a classical measure of non-Gaussianity is kurtosis or the fourth-order cumulant. The kurtosis of $\mathbf{y}$ (zero-mean and unit-variance) is classically defined by

$$
\operatorname{kurt}(\mathbf{y})=E\left\{\mathbf{y}^{4}\right\}-3\left(E\left\{\mathbf{y}^{2}\right\}\right)^{2}
$$

The main reason of kurtosis as a widely used measure of independence is its computational and theoretical simplicity. Computationally, kurtosis can be estimated simply by using the fourth moment of the sample data. Theoretically, kurtosis has good performances for linear transform:

$$
\begin{gathered}
\operatorname{kurt}\left(\mathbf{y}_{1}+\mathbf{y}_{2}\right)=\operatorname{kurt}\left(\mathbf{y}_{1}\right)+\operatorname{kurt}\left(\mathbf{y}_{2}\right) \\
\operatorname{kurt}(\alpha \mathbf{y})=\alpha^{4} \operatorname{kurt}(\mathbf{y})
\end{gathered}
$$

where $\alpha$ is a scalar.

Another and very important measure of non-Gaussianity is given by negentropy, which is based on the information theoretical quantity of entropy. The more "random" the variable is, the larger its entropy will be. Entropy is normally defined as

$$
H(\mathbf{y})=-\int f(\mathbf{y}) \log f(\mathbf{y}) d \mathbf{y}
$$

where $f(\mathbf{y})$ is the density of a random vector $\mathbf{y}$.

Negentropy is used to obtain an effective measure of nonGaussianity, and can be defined as follows:

$$
J(\mathbf{y})=H\left(\mathbf{y}_{\text {gauss }}\right)-H(\mathbf{y})
$$

where $\mathbf{y}_{\text {gauss }}$ is a Gaussian variable of the same covariance matrix as $\mathbf{y}$.

The advantage of using negentropy as an effective measure of non-Gaussianity is that it is well justified by statistical theory. However, the estimation of negentropy is difficult, and therefore some approximations have to be 
used to simplify the calculation. The classical method of approximating negentropy is using higher-order moments:

$$
J(\mathbf{y}) \approx \frac{1}{12} E\left\{\mathbf{y}^{3}\right\}^{2}+\frac{1}{48} \operatorname{kurt}(\mathbf{y})^{2}
$$

\subsection{Efficient ICA Algorithm}

2.3.1. Centering. The most basic and necessary process before an effective source separation is to center $\mathbf{x}$ :

$$
\mathbf{x}=\mathbf{x}-\operatorname{mean}(\mathbf{x})
$$

2.3.2. Whitening. One popular method for whitening is to use the eigenvalue decomposition (EVD) of the covariance matrix $E\left\{\mathbf{x} \mathbf{x}^{T}\right\}=\mathbf{E D E}^{T}$, where $\mathbf{E}$ is the orthogonal matrix of eigenvectors of $E\left\{\mathbf{x x}^{T}\right\}$ and $\mathbf{D}$ is the diagonal matrix of its eigenvalues, $\mathbf{D}=\operatorname{diag}\left(d_{1}, \ldots d_{n}\right)$ :

$$
\widetilde{\mathbf{x}}=\mathbf{E D}^{-1 / 2} \mathbf{E}^{T} \mathbf{x}
$$

where $\mathbf{D}^{-1 / 2}=\operatorname{diag}\left(d_{1}^{-1 / 2}, \ldots, d_{n}^{-1 / 2}\right)$.

\subsubsection{Fixed Point Iteration Scheme for One Component}

(1) Choose an initial weight vector $\mathbf{w}$.

(2) Let $\mathbf{w}^{+}=E\left\{\mathbf{x} g\left(\mathbf{w}^{T} \mathbf{x}\right)\right\}-E\left\{g^{\prime}\left(\mathbf{w}^{T} \mathbf{x}\right)\right\} \mathbf{w}$, where $g$ is the derivatives of the nonquadratic function and normally has

$$
g_{1}(\mathbf{u})=\tanh (a \mathbf{u}), \quad g_{2}(\mathbf{u})=\mathbf{u} \exp \left(-\frac{\mathbf{u}^{2}}{2}\right)
$$

where $1 \leq a \leq 2$ is a suitable constant.

(3) Let $\mathbf{w}=\mathbf{w}^{+} /\left\|\mathbf{w}^{+}\right\|$.

(4) If not converged, go back to (2).

\subsubsection{Fixed Point Iteration Scheme for Several Components}

(1) Let $\mathbf{w}_{p+1}=\mathbf{w}_{p+1}-\sum_{j=1}^{p} \mathbf{w}_{p+1}^{T} \mathbf{w}_{j} \mathbf{w}_{j}$.

(2) Let $\mathbf{w}_{p+1}=\mathbf{w}_{p+1} / \sqrt{\mathbf{w}_{p+1}^{T} \mathbf{w}_{p+1}}$.
Comparing the separating vector $\mathbf{w}$ for one component, the separating matrix $\mathbf{W}=\left(\mathbf{w}_{1}, \ldots, \mathbf{w}_{n}\right)^{T}$ for several components can be obtained as follows:

$$
\begin{gathered}
\mathbf{W}=\frac{\mathbf{W}}{\sqrt{\left\|\mathbf{W W}^{T}\right\|}}, \\
\mathbf{W}=\frac{3}{2} \mathbf{W}-\frac{1}{2} \mathbf{W} \mathbf{W}^{T} \mathbf{W} .
\end{gathered}
$$

2.4. Source Identification. For mechanical systems, some information of the sources normally can be obtained by theory study or instructions. Therefore, waveform correlation can be used to identify the source information by a correlation analysis between the sources and the separated signals. For discrete signals s and $\mathbf{y}$, waveform correlation coefficient $\rho_{W}$ is defined as

$$
\rho_{W}=\frac{\max R_{\mathrm{sy}}(k)}{\sqrt{\max R_{\mathrm{ss}}(k) \times \max R_{\mathrm{yy}}(k)}}, \quad 0 \leq k \leq T,
$$

where $R_{\mathbf{s y}}(k)=\sum_{i=0}^{T-k-1} \mathbf{s}(i+k) \mathbf{y}(i), R_{\mathbf{s s}}(k)=\sum_{i=0}^{T-k-1} \mathbf{s}(i+$ $k) \mathbf{s}(i), R_{\mathbf{y y}}(k)=\sum_{i=0}^{T-k-1} \mathbf{y}(i+k) \mathbf{y}(i), \forall k \geq 0$, and $T$ is the data length.

\section{Numerical Case Study}

In this section, typical vibration and acoustical signals of mechanical systems are artificially generated to test the separation performance of the ICA algorithm based on negentropy. Since acoustical signals transmit from the sources to the measured points through air, the mixing mode of all the acoustical source signals tends to be a linear superposition. Furthermore, the generating mechanism of different sources is far from each other. Therefore, all the sources are considered to be linearly mixed and independent of each other.

The given source signals are typical vibration and acoustical signals of mechanical systems: signal $\mathbf{s}_{1}(t)$ is a sinusoidal wave that simulates a vibration signal of rotational equipment; signal $\mathbf{s}_{2}(t)$ is a periodic wave of oscillating attenuation that simulates mechanical shocks; signal $\mathbf{s}_{3}(t)$ is a periodic wave that simulates amplitude modulation; signal $\mathbf{s}_{4}(t)$ is a periodic wave that simulates frequency modulation; and signal $\mathbf{s}_{5}(t)$ is a white noise that simulates noises produced by environment and structural transmission. The generating functions of sources are as follows:

$$
\mathbf{S}(t)=\left[\begin{array}{l}
\mathbf{s}_{1}(t) \\
\mathbf{s}_{2}(t) \\
\mathbf{s}_{3}(t) \\
\mathbf{s}_{4}(t) \\
\mathbf{s}_{5}(t)
\end{array}\right]=\left[\begin{array}{c}
\sin (15 \pi t) \\
\sum \sin (1000(t-0.1 n-0.02)) \exp \left(-\frac{150(t-0.1 n-0.02)}{2}\right) u(t-0.1 n-0.02) \\
\sin (10 \pi t) \cos (150 \pi t) \\
\sin (6 \pi \cos (6 \pi t)) \\
n(t)
\end{array}\right],
$$

where $u(t)$ is a step function. 

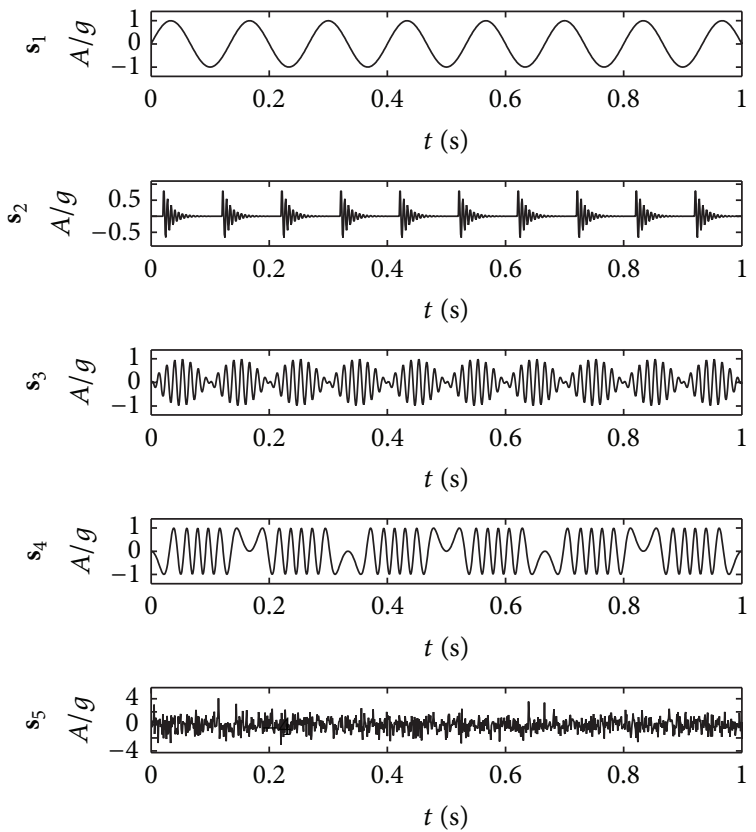

FIGURE 1: Waveforms of the source signals.

Generally, the measured acoustical signals are mixed signals of all the sources with noises. Furthermore, as the acoustical signals transmit from the sources to the measuring points through air, linear superposition is applied to artificially produce the mixed signals. The mixing matrix $\mathbf{A}$ is randomly generated as the following matrix:

$$
\mathbf{A}=\left[\begin{array}{ccccc}
0.23 & -0.58 & 0.73 & -0.81 & 0.95 \\
0.28 & -0.89 & 0.49 & -0.75 & 0.97 \\
0.56 & 0.15 & -0.72 & 0.58 & 0.99 \\
-0.38 & -0.52 & 0.45 & 0.61 & 0.99 \\
-0.48 & 0.38 & 0.84 & -0.51 & 0.98 \\
0.88 & -0.61 & 0.54 & -0.20 & 0.95
\end{array}\right]
$$

The waveforms of the source signals and the mixed signals are shown in Figures 1 and 2. Obviously, all the source signals have typical waveform features. However, it is very difficult to directly identify the features of the mixed signals as the complicated waveforms. Therefore, generally signal processing methods are required to help to extract the desired features clearly. In this numerical case study, the ICA algorithm based on negentropy is applied to separate the mixed signals into 5 independent components, and then these separated components are identified by the correlation analysis.

With the ICA algorithm, 6 mixed signals are separated into 5 independent components, and the waveforms of these independent components are shown in Figure 3. Comparing Figure 3 with Figure 1, obviously the waveform features of each source signal are well separated. Waveform correlation analysis is used to quantitatively evaluate the separation performances of the ICA algorithm and identify the sources,
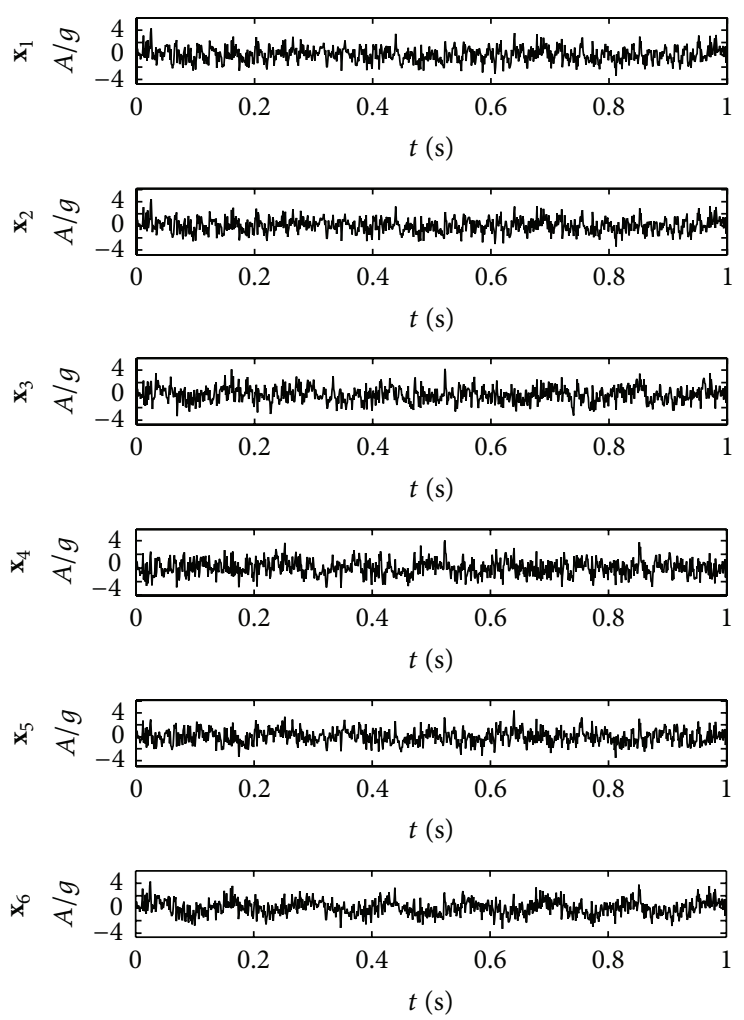

Figure 2: Waveforms of the mixed signals.

and the waveform correlation coefficients $\rho_{W}$ are shown in the following matrix:

$$
\Omega_{W}=\left[\begin{array}{lllll}
\mathbf{0 . 9 9} & 0.01 & 0.04 & 0.00 & 0.04 \\
0.01 & \mathbf{0 . 9 9} & 0.03 & 0.17 & 0.12 \\
0.03 & 0.03 & \mathbf{0 . 9 9} & 0.01 & 0.09 \\
0.00 & 0.15 & 0.01 & \mathbf{0 . 9 9} & 0.03 \\
0.05 & 0.12 & 0.09 & 0.04 & \mathbf{0 . 9 9}
\end{array}\right] .
$$

Each correlation coefficient $\rho_{i j}$ in $\Omega_{W}=\left\{\rho_{i j}\right\}$ indicates the similarity between the separated component $i$ and the source $j$. From the correlation matrix $\Omega_{W}=\left\{\rho_{i j}\right\}$, the elements in the diagonal show the correlation coefficients between the separated components and related source signals: the correlation coefficient of $\mathbf{s}_{1}\left(\mathbf{s}_{2}, \mathbf{s}_{3}, \mathbf{s}_{4}\right.$, and $\left.\mathbf{s}_{5}\right)$ and related separated component $1(2,3,4$, and 5$)$ is $\mathbf{0 . 9 9}$, which indicates that all the source information of $\mathbf{s}_{1}\left(\mathbf{s}_{2}, \mathbf{s}_{3}, \mathbf{s}_{4}\right.$, and $\left.\mathbf{s}_{5}\right)$ has been completely separated from the mixed signals by the ICA algorithm. The high correlation coefficients indicate that the ICA algorithm can effectively separate the mixed signals into independent components for the given sources and linearly mixed mode. However, the correlation coefficients between the independent components and the unrelated sources are all less than 0.17, which indicates that all the separated components have good independent properties. Therefore, the ICA algorithm based on negentropy is applied to extract the source information from the measured acoustical signals from real mechanical systems, and the separated information 

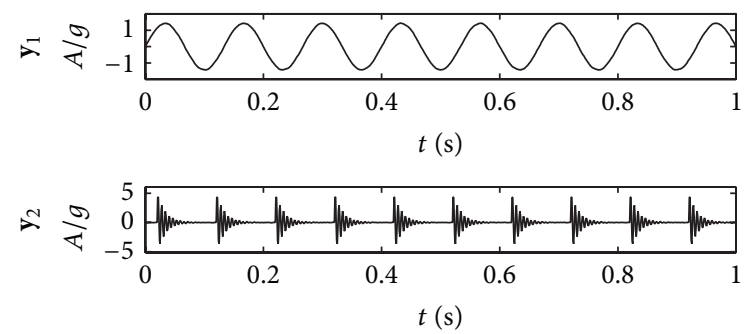

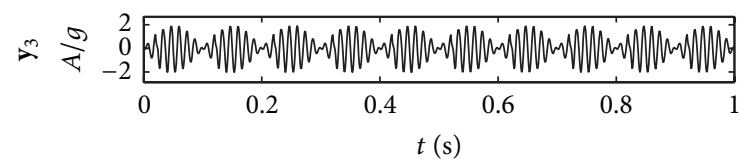
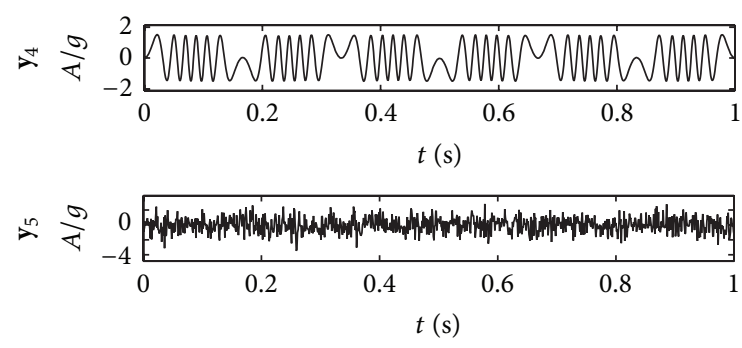

FIGURE 3: Separated components by DSS.

is used to validate the effectiveness of ICA algorithm and trace the source information hidden in the measured signals.

\section{Experimental Study}

4.1. Introductions of the Test Bed. A test bed of a mechanical system with shell structures is constructed to test the separation performances of the ICA algorithm based on negentropy, which is composed of four components: an end cover, a shell, two clapboards, and supports. Rubber air springs are used to support the whole test bed and reduce the effects of the ground vibrations and environmental noises. Three acoustical sources are designed in the test bed: two loudspeakers controlled by the signal generators and one motor controlled by the frequency converter. The structure of the test bed is shown in Figure 4.

Six sound pressure sensors are used to measure the acoustical signals, and they are located in six directions of the test bed with a distance of 500 millimeters. HBM Gen2i data acquisition system is applied to collect the acoustical data from these six sensors. The framework of the measuring system is shown in Figure 5, and the test parameters are shown in Table 1.

4.2. Acoustical Signals of the Test Bed. The acoustical signals measured by the six sound pressure sensors are the mixed signals of all the sources as they are working together. In the experimental study, the acoustical signals from sensors 1,4 , and 6 are used as the mixed signals so as to satisfy the assumptions of ICA: the number of the mixed signals should be no less than the number of the source signals. Furthermore, the directions of these sensors represent a diversified mixing mode of all the sources.

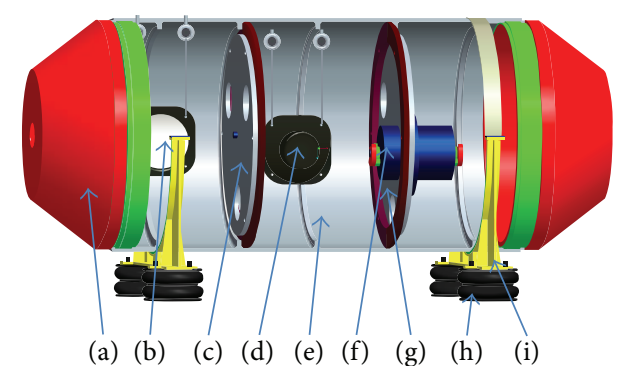
(a) End cover
(b) Loudspeaker I
(f) Motor
(c) Left clapboard
(g) Right clapboard
(d) Loudspeaker II
(h) Rubber springs
(i) Supports

(e) Shell

Figure 4: The structure of the test bed.

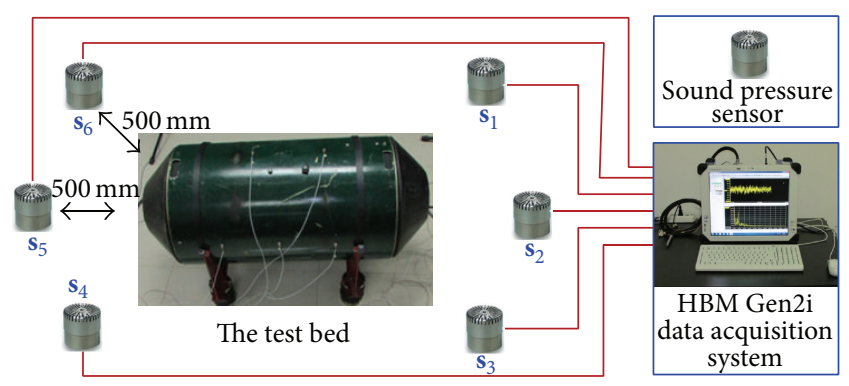

FIgURE 5: The framework of the measuring system.

TABLE 1: The test parameters of the measuring system.

\begin{tabular}{lc}
\hline Parameters & Values and units \\
\hline Sound pressure sensors & 6 \\
HBM Gen2i data acquisition system & 1 \\
Test bed with shell structure & 1 \\
Sampling frequency & $10240 \mathrm{~Hz}$ \\
Sampling length & 10 seconds \\
Rotational speed of motor & $1800 \mathrm{r} / \mathrm{min}(20 \mathrm{~Hz})$ \\
Frequency of Loudspeaker I & $1750 \mathrm{~Hz}$ \\
Frequencies of Loudspeaker II & 500,1700, and $2000 \mathrm{~Hz}$ \\
\hline
\end{tabular}

The waveforms and spectrums of the mixed signals are shown in Figures 6 and 7, respectively. From waveforms in Figure 6, it is difficult to identify the waveform features of the source signals except some periodic waves, which indicates that the waveforms of the mixed signals are complicated, and normally signal processing method is required to extract the desired features. From spectrums in Figure 7, some major components of $20,1100,1700,1750$, and $2000 \mathrm{~Hz}$ are clear, which represent the characteristic features of the sources as the experimental settings. Generally, the independent information of the source signals cannot be directly identified from the measured mixed signals as each mixed signal contains all the major components of the source signals. 

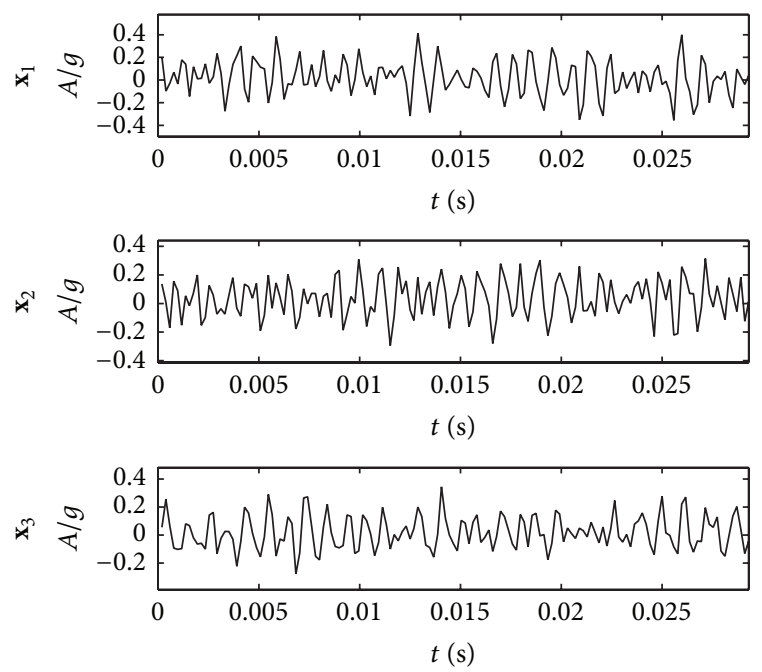

FIGURE 6: Waveforms of the mixed signals.
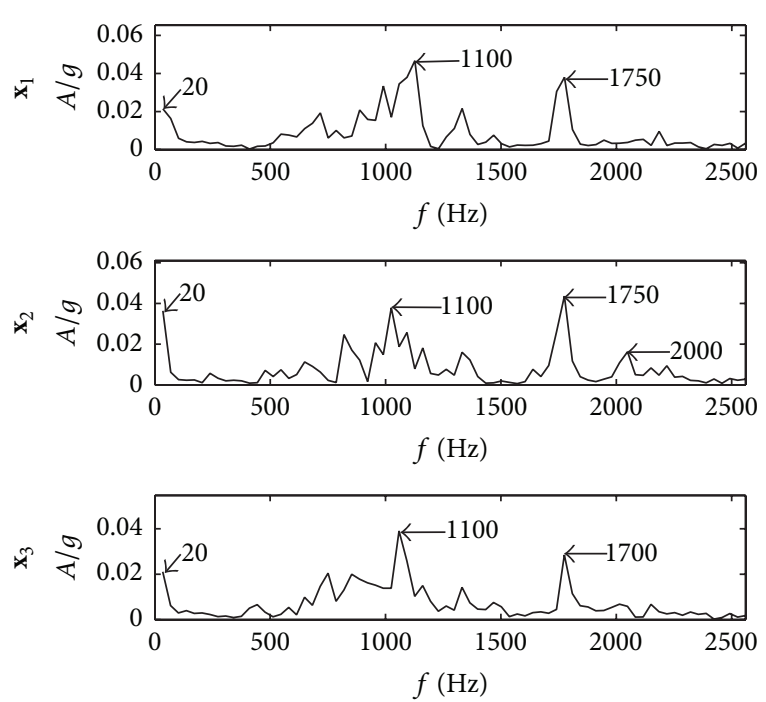

FIGURE 7: Spectrums of the mixed signals.

4.3. Acoustical Source Separation. The ICA algorithm based on negentropy is applied to separate the mixed signals into independent components, and 3 independent components are extracted from the given mixed signals. The waveforms and spectrums of the independent components are shown in Figures 8 and 9, respectively. Figure 8 clearly shows that the waveform of the separated component $\mathbf{y}_{1}$ has typically periodic and amplitude-modulation features, and the basic components are sine waves, which are normally caused by the eccentric vibration of mechanical systems. The spectrums in Figure 9 also show that the separated component $\mathbf{y}_{1}$ has major components of 20 and $2000 \mathrm{~Hz}$. The waveform of the separated component $\mathbf{y}_{2}$ has typical features of sine waves, and its spectrum also clearly shows the major component of $1750 \mathrm{~Hz}$. The waveform of the separated component $\mathbf{y}_{3}$ has an obvious feature of sine waves with a periodic amplitude-modulation,
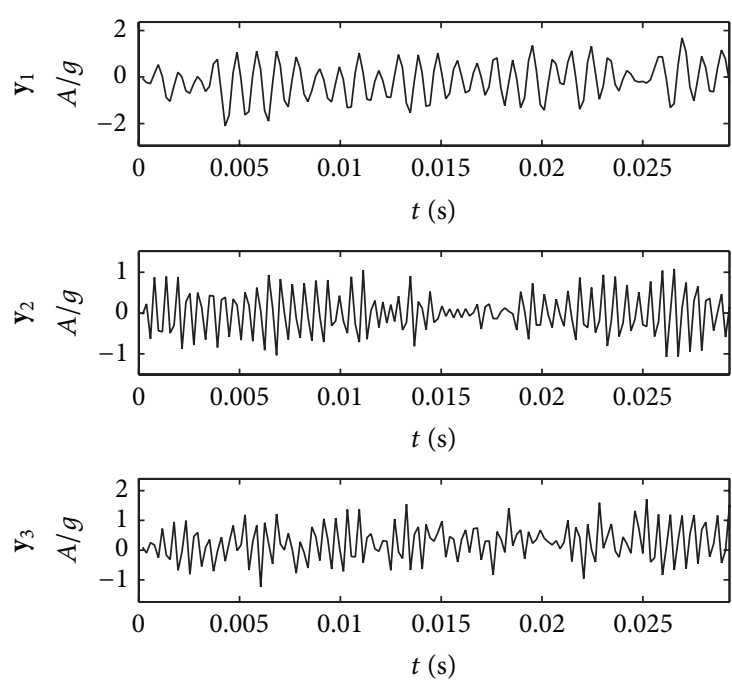

FIGURE 8: Waveforms of separated components.
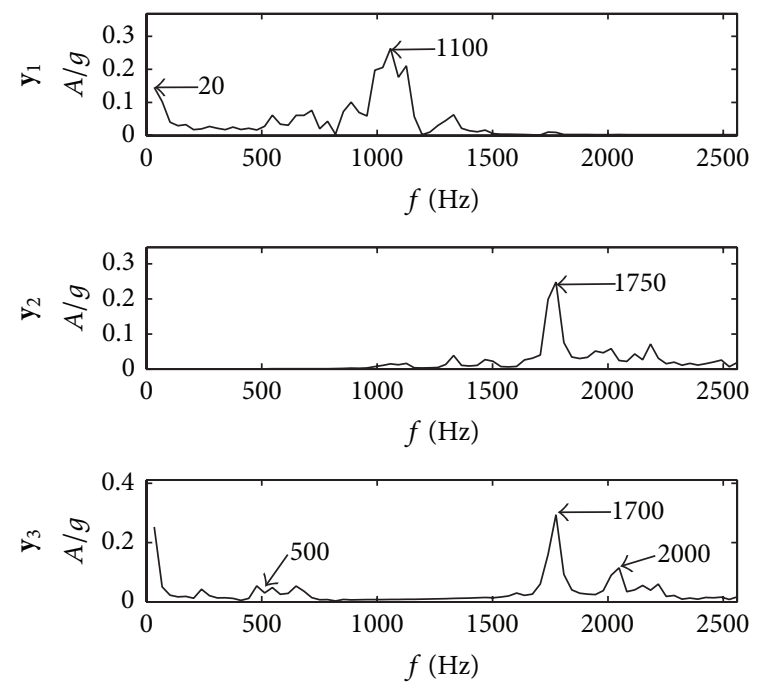

FIGURE 9: Spectrums of separated components.

and its spectrum contains three major components of 500, 1700 , and $2000 \mathrm{~Hz}$.

Comparing the spectrums of the separated components with the parameters of the experimental settings, it can be speculated that the separated component $\mathbf{y}_{1}$ represents the typical feature of the source 1 from a motor, while the separated components $\mathbf{y}_{2}$ and $\mathbf{y}_{3}$ represent the typical features of the sources 2 and 3 from Loudspeakers I and II, respectively. However, this is just based on the parameters of the experimental settings and it is still not a convincing and reliable source tracing method.

4.4. Acoustical Source Tracing and Validation. To intelligently trace the sources and validate the effectiveness of the ICA algorithm in real mechanical systems, acoustical source signals are measured independently by the closest sensors in the condition that only one source is working with the given 

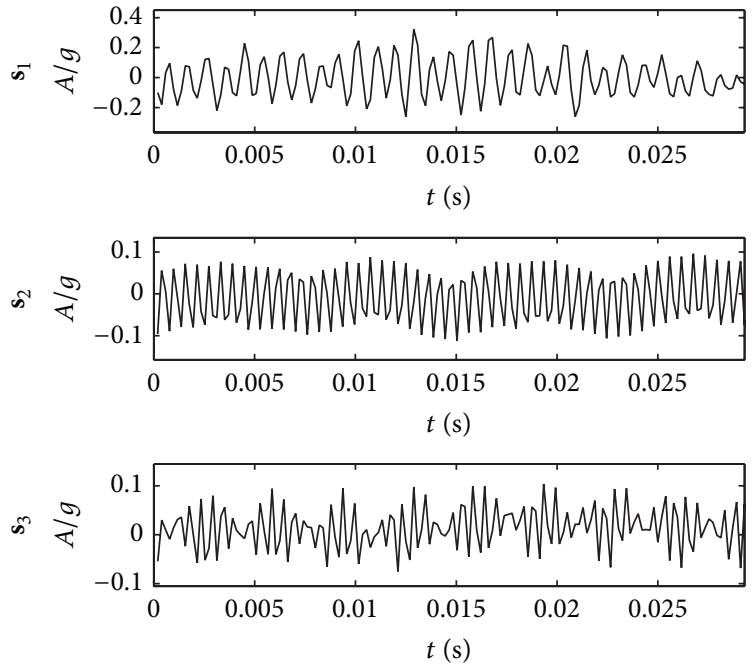

FIGURE 10: Waveforms of the source signals.
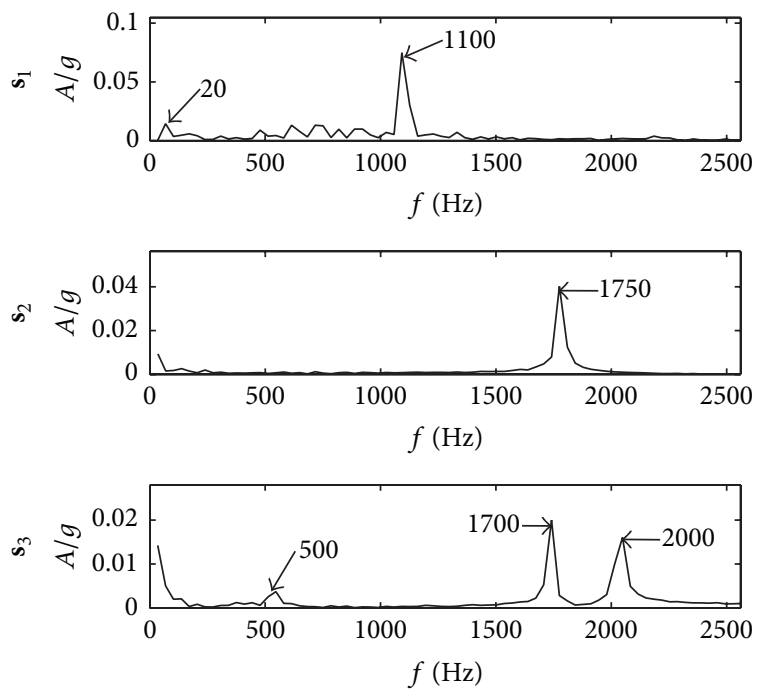

FIGURE 11: Spectrums of the source signals.

parameters. The independent source waveforms from sensors 1,4 , and 6 in the condition that only the motor, Loudspeaker I, or Loudspeaker II is working with the given experimental settings are shown in Figure 10, and their spectrums are shown in Figure 11.

Comparing the waveforms and spectrums of the source signals with those of the independent components, the waveforms of the independent components are very similar to those of the related source signals: the independent component $\mathbf{y}_{1}$ and the source signal $\mathbf{s}_{1}$ have a typical feature of sine waves with an uncertainty amplitude modulation, which can be caused by the eccentric vibration of the motor, and the uncertainty amplitude modulation can be caused by the rubbing; the independent component $\mathbf{y}_{2}$ and the source signal $\mathbf{s}_{2}$ have the same major component of $1750 \mathrm{~Hz}$, which agrees with the experimental settings; the independent component $\mathbf{y}_{3}$ and the source signal $\mathbf{s}_{3}$ have three same major components of 500,1700 , and $2000 \mathrm{~Hz}$ as the experimental settings. Therefore, all the major components of the source signals can be effectively traced by the ICA algorithm based on negentropy.

To quantitatively and intelligently trace the sources, the waveform correlation analysis is used to evaluate the similarity between independent components and the sources. All the independent components are made a correlation analysis with all the sources, and the correlation coefficients are listed in the correlation matrix $\Omega_{W}$ :

$$
\Omega_{W}=\left[\begin{array}{lll}
\mathbf{0 . 9 0} & 0.07 & 0.06 \\
0.05 & \mathbf{0 . 7 6} & 0.08 \\
0.10 & 0.16 & \mathbf{0 . 8 5}
\end{array}\right] .
$$

The correlation matrix $\Omega_{W}$ shows that the correlation coefficients between the independent components and the related sources are $\mathbf{0 . 9 0}, \mathbf{0 . 7 6}$, and $\mathbf{0 . 8 5}$, which indicate high correlation coefficients and high similarity between the independent components and the related sources (Liu et al. [25] obtained waveform correlation coefficients of $0.77 \pm$ 0.03 for ECG signals with noises, and Farina et al. [26] obtained correlation coefficients of $0.70 \pm 0.09$ for nonstationary surface myoelectric signals), while the correlated coefficients between the independent components and the unrelated sources are less than 0.16 , which indicates that all the independent components and all the sources have good independence property. Therefore, a threshold $\gamma$ can be set as $\gamma \in(0.16,0.76)$ (in practice $\gamma \in(0.50,0.70)$ ) to intelligently identify and trace the acoustical sources, and the high correlation coefficients between the independent components and the related sources validate the effectiveness of the ICA algorithm based on negentropy in source separation and acoustical source tracing.

\section{Conclusions}

This paper presents the fundamental theory of blind source separation and key principals of independent component analysis and validates the effectiveness of the ICA algorithm based on negentropy according to a numerical case study and an experimental study on a mechanical system with shell structures. The experimental study indicates that the acoustical sources can be effectively separated and intelligently traced.

In the numerical case study, five typical acoustical source signals of mechanical systems are effectively separated from six linearly mixed signals, and the correlation coefficients between the independent components and the related source signals are all more than 0.99 , which indicates a complete and highly effective source separation of the ICA algorithm for the given mixed signals while in the experimental study on a mechanical system with shell structures, the correlation coefficients between the independent components and the related source signals are all more than 0.76 , which also reveals an effective acoustical source separation. If artificially giving a threshold $\gamma \in(0.50,0.70)$ for the correlation coefficients, all the acoustical sources can be intelligently identified and traced. 
This work can provide pure source information for machinery condition monitoring and fault diagnosis, and the independent source information can also be of benefit for noise identification, reduction, and control.

\section{Conflict of Interests}

The authors declare that there is no conflict of interests regarding the publication of this paper.

\section{Acknowledgments}

This work was supported by the projects of National Nature Science Foundation of China (no. 51305329, 51035007), the China Postdoctoral Science Foundation (nos. 2013M532032 and 2014T70911), the Doctoral Foundation of Education Ministry of China (no. 20130201120040), and the Shaanxi Postdoctoral Scientific Research Project.

\section{References}

[1] F. X. Xin, T. J. Lu, and C. Q. Chen, "Sound transmission through simply supported finite double-panel partitions with enclosed air cavity," Journal of Vibration and Acoustics, vol. 132, no. 1, 2010.

[2] C. Z. Huang and S. Nutt, "An analytical study of sound transmission through unbounded panels of functionally graded materials," Journal of Sound and Vibration, vol. 330, no. 6, pp. 1153-1165, 2011.

[3] T. Bravo, C. Maury, and C. Pinhede, "Sound absorption and transmission through flexible micro-perforated panels backed by an air layer and a thin plate," Journal of the Acoustical Society of America, vol. 131, no. 5, pp. 3853-3863, 2012.

[4] Z. C. Qian, D. Q. Chang, B. L. Liu, and K. Liu, "Prediction of sound transmission loss for finite sandwich panels based on a test procedure on beam elements," Journal of Vibration and Acoustics, Transactions of the ASME, vol. 135, no. 6, 2013.

[5] H. Posson, H. Bériot, and S. Moreau, "On the use of an analytical cascade response function to predict sound transmission through an annular cascade," Journal of Sound and Vibration, vol. 332, no. 15, pp. 3706-3739, 2013.

[6] B. C. Lee and S. R. Kim, "Effect of structure on sound absorption and sound transmission loss of composite sheet," Advanced Composite Materials, vol. 23, no. 4, pp. 319-325, 2014.

[7] N. Chandra, S. Raja, and K. V. N. Gopal, "Vibro-acoustic response and sound transmission loss analysis of functionally graded plates," Journal of Sound and Vibration, vol. 333, no. 22, pp. 5786-5802, 2014.

[8] C. Jutten and J. Herault, "Blind separation of sources, part I: an adaptive algorithm based on neuromimetic architecture," Signal Processing, vol. 24, no. 1, pp. 1-10, 1991.

[9] A. Belouchrani, K. Abed-Meraim, J.-F. Cardoso, and E. Moulines, "A blind source separation technique using secondorder statistics," IEEE Transactions on Signal Processing, vol. 45, no. 2, pp. 434-444, 1997.

[10] P. Comon, "Independent component analysis, a new concept?" Signal Processing, vol. 36, no. 3, pp. 287-314, 1994.

[11] A. Hyvärinen, "Fast and robust fixed-point algorithms for independent component analysis," IEEE Transactions on Neural Networks, vol. 10, no. 3, pp. 626-634, 1999.
[12] A. Hyvärinen and E. Oja, "Independent component analysis: algorithms and applications," Neural Networks, vol. 13, no. 4-5, pp. 411-430, 2000.

[13] D.-M. Tsai and S.-C. Lai, "Independent component analysisbased background subtraction for indoor surveillance," IEEE Transactions on Image Processing, vol. 18, no. 1, pp. 158-167, 2009.

[14] A. Hyvärinen, P. Ramkumar, L. Parkkonen, and R. Hari, "Independent component analysis of short-time Fourier transforms for spontaneous EEG/MEG analysis," NeuroImage, vol. 49, no. 1, pp. 257-271, 2010.

[15] J. Gao, X. Zhu, and A. K. Nandi, “Independent component analysis for multiple-input multiple-output wireless communication systems," Signal Processing, vol. 91, no. 4, pp. 607-623, 2011.

[16] J. Ji, "Robust approach to independent component analysis for SAR image analysis," IET Image Processing, vol. 6, no. 3, pp. 284291, 2012

[17] R. Rodríguez-Rocha, F. J. Rivero-Angeles, and E. GómezRamírez, "Application of the baseline stiffness method for damage detection of an RC building without baseline modal parameters utilizing the independent component analysis for modal extraction," International Journal of Structural Stability and Dynamics, vol. 13, no. 3, Article ID 1250074, 2013.

[18] J. Antoni, "Blind separation of vibration components: principles and demonstrations," Mechanical Systems and Signal Processing, vol. 19, no. 6, pp. 1166-1180, 2005.

[19] W. Cheng, S. Lee, Z. S. Zhang, and Z. J. He, "Independent component analysis based source number estimation and its comparison for mechanical systems," Journal of Sound and Vibration, vol. 331, no. 23, pp. 5153-5167, 2012.

[20] W. Cheng, Z. J. He, and Z. S. Zhang, "A comprehensive study of vibration signals for a thin shell structure using enhanced independent component analysis and experimental validation," Journal of Vibration and Acoustics, vol. 136, no. 4, Article ID 041011, 2014.

[21] W. Cheng, Z. S. Zhang, S. Lee, and Z. J. He, "Source contribution evaluation of mechanical vibration signals via enhanced independent component analysis," Transactions of the ASME, Journal of Manufacturing Science and Engineering, vol. 134, no. 2, Article ID 021014, 2012.

[22] P. Henriquez, J. B. Alonso, M. A. Ferrer, and C. M. Travieso, "Review of automatic fault diagnosis systems using audio and vibration signals," IEEE Transactions on Systems, Man, and Cybernetics: Systems, vol. 44, no. 5, pp. 642-652, 2014.

[23] P. Comon, C. Jutten, and J. Herault, "Blind separation of sources. Part II: problems statement," Signal Processing, vol. 24, no. 1, pp. 11-20, 1991.

[24] E. Sorouchyari, "Blind separation of sources. Part III. Stability analysis," Signal Processing, vol. 24, no. 1, pp. 21-29, 1991.

[25] H. Liu, C. Q. Chang, K. D. K. Luk, and Y. Hu, "Comparison of blind source separation methods in fast somatosensory-evoked potential detection," Journal of Clinical Neurophysiology, vol. 28, no. 2, pp. 170-177, 2011.

[26] D. Farina, C. Févotte, C. Doncarli, and R. Merletti, "Blind separation of linear instantaneous mixtures of nonstationary surface myoelectric signals," IEEE Transactions on Biomedical Engineering, vol. 51, no. 9, pp. 1555-1567, 2004. 

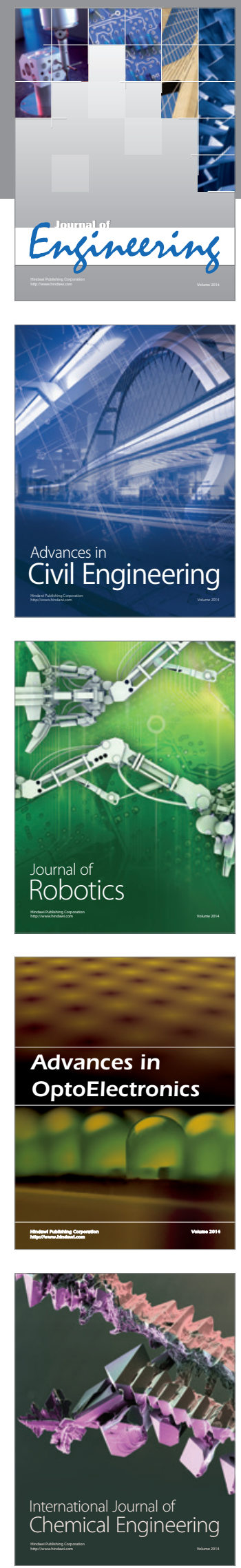

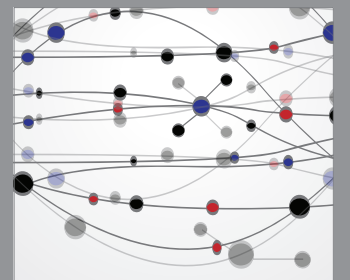

The Scientific World Journal
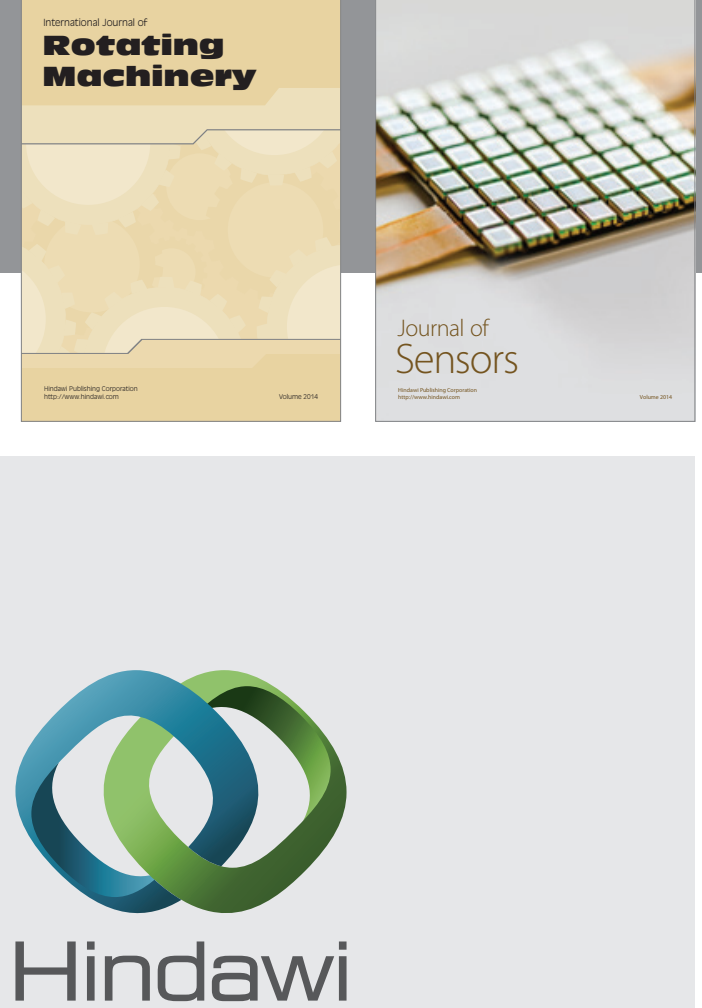

Submit your manuscripts at http://www.hindawi.com
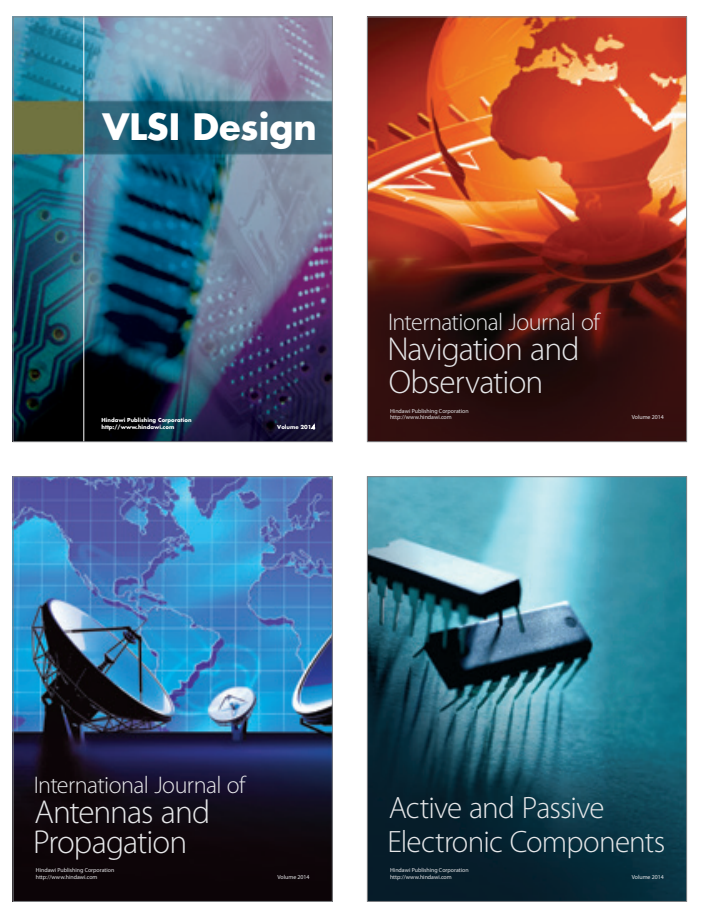
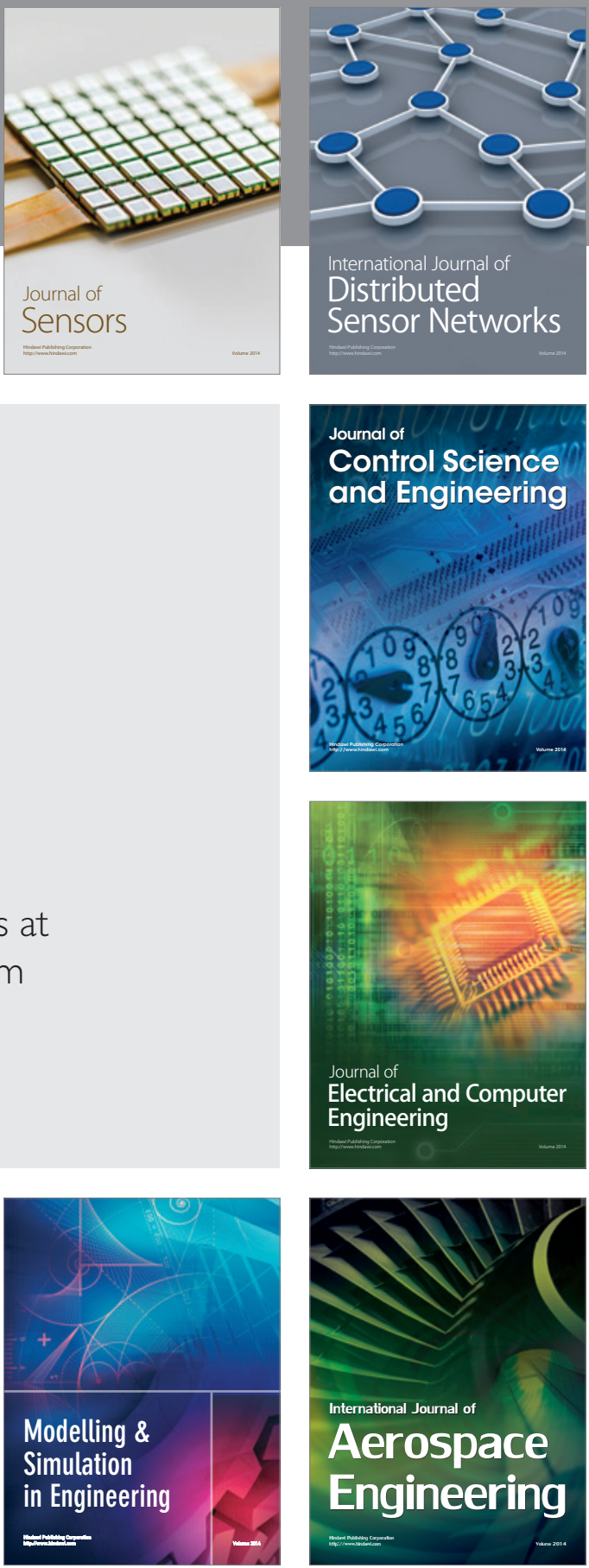

Journal of

Control Science

and Engineering
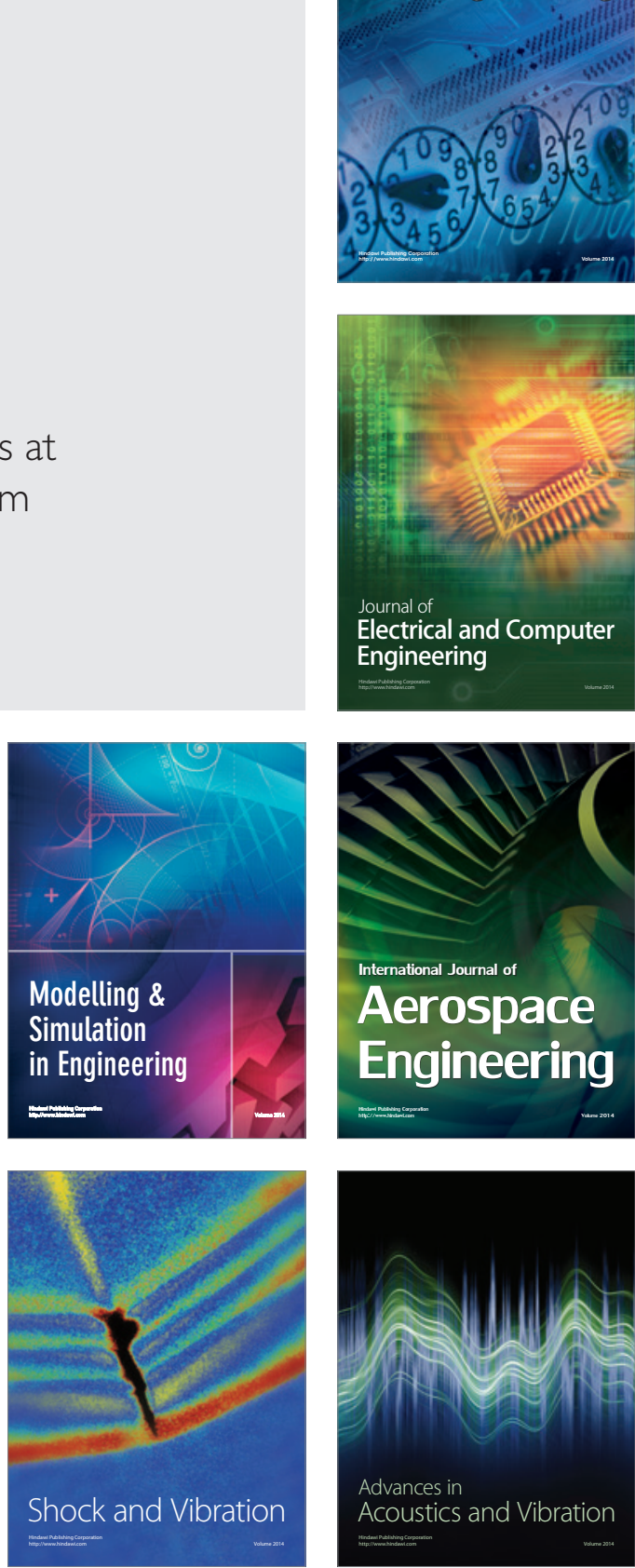